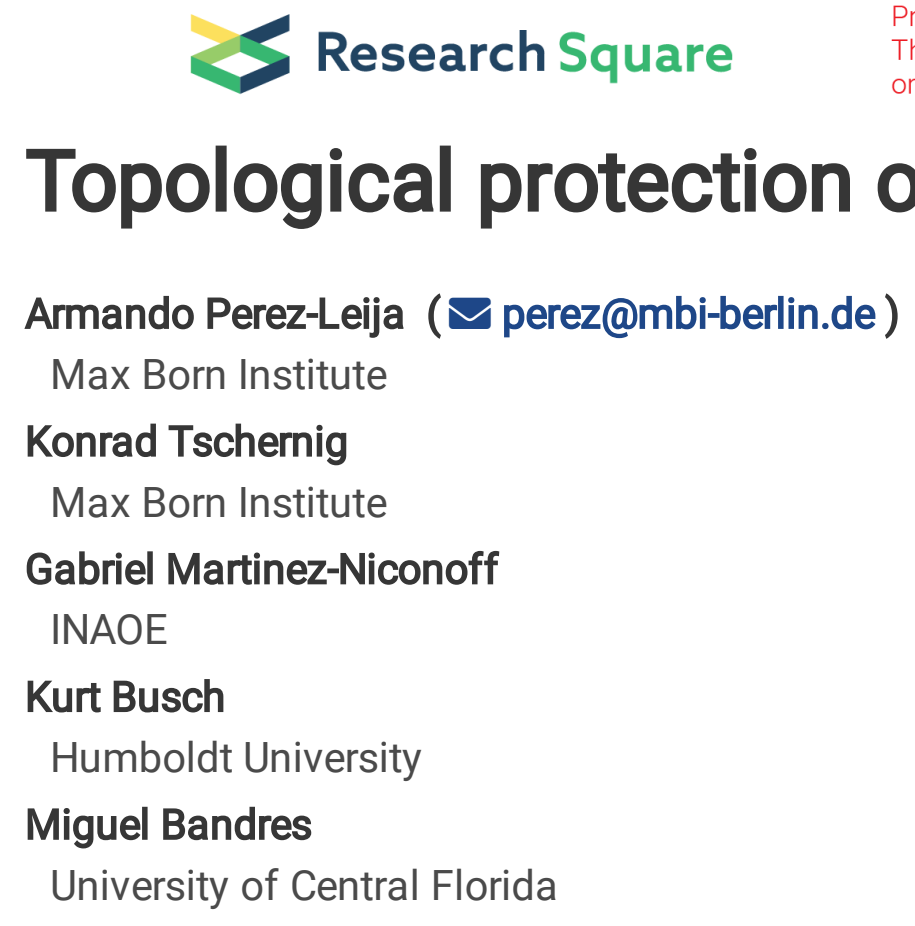

\author{
Armando Perez-Leija ( $\sim$ perez@mbi-berlin.de) \\ Konrad Tschernig \\ Max Born Institute \\ Gabriel Martinez-Niconoff \\ INAOE \\ Kurt Busch \\ Humboldt University \\ Miguel Bandres \\ University of Central Florida
}

Max Born Institute

\title{
Topological protection of partially coherent light
}

\section{Article}

Keywords:

Posted Date: February 1st, 2022

DOI: https://doi.org/10.21203/rs.3.rs-1183022/v1

License: (c) (i) This work is licensed under a Creative Commons Attribution 4.0 International License.

Read Full License 


\section{Topological protection of partially coherent light}

Konrad Tschernig, ${ }^{1,2, *}$ Gabriel Martinez-Niconoff, ${ }^{3}$ Kurt Busch, ${ }^{1,2}$

Miguel A. Bandres, ${ }^{4, \dagger}$ and Armando Perez-Leija ${ }^{1,2, \ddagger}$

${ }^{1}$ Max-Born-Institut, Max-Born-Straße 2A, 12489 Berlin, Germany

${ }^{2}$ Humboldt-Universität zu Berlin, Institut für Physik,

AG Theoretische Optik 8 Photonik, Newtonstraße 15, 12489 Berlin, Germany

${ }^{3}$ Coordinación de Óptica, Instituto Nacional de Astrofísica, Óptica, y Electrónica, Luis Enrique Erro No 1, 72840, Tonantzintla, Puebla, México

${ }^{4}$ CREOL, The College of Optics and Photonics, University of Central Florida, Orlando, FL 32816-2700, USA

(Dated: December 10, 2021)

Topological physics exploits concepts from geometry and topology to implement systems capable of guiding waves in an unprecedented fashion. These ideas have led to the development of photonic topological insulators, which are optical systems whose eigenspectral topology allows the creation of light states that propagate along the edge of the system without any coupling into the bulk or backscattering even in the presence disorder. Indeed, topological protection is a fully coherent effect, and it is not clear to what extent topological effects endure when the wavefronts become partially coherent. Here, we study the interplay of topological protection and the degree of spatial coherence of classical light propagating in disordered photonic topological insulators. Our results reveal the existence of a well-defined spectral window in which partially coherent light is topologically protected. This opens up the design space to a wider selection of light sources, possibly yielding smaller, cheaper and more robust devices based on the topological transport of light. 


\section{Introduction}

Topological insulators are materials in which the topology of the underlying energy spectra fully disallows the energy transport into the bulk and keeps it circulating along the edges ${ }^{1}$. Topological insulators were discovered in the context of single-particle condensed matter physics ${ }^{2}$, and as such they have inspired the quest for topological phases in classical-wave systems ${ }^{3}$, including microwaves ${ }^{1}$, photonic $^{4-9}$, and acoustic systems ${ }^{10,11}$. At the forefront, photonics has become one of the most prolific and fertile grounds to study and observe topological effects using so-called photonic topological insulators ${ }^{12}$. Indeed, optics has recently witnessed the experimental demonstration of Floquet topological insulators ${ }^{4}$, the quantum Hall effect ${ }^{6}$, topological lasers ${ }^{13,14}$, and aperiodic topological systems in which the topology is induced by disorder (e.g., topological quasicrystals ${ }^{15}$, topological Anderson insulators ${ }^{16}$, topological insulators in fractal lattices $\left.{ }^{17}\right)$. Notably, in these latter systems the aperiodicity and disorder have a dramatic impact on the physical properties, not only modifying the topology of the spectra but also creating a topological order in otherwise topologically trivial systems. Concurrently, in the context of quantum optics there have emerged very appealing theoretical and experimental studies of topological protection of entangled states of several photons ${ }^{18-22}$.

Indeed, topological protection is a fully coherent process and it is not clear to what extent topological effects endure when the excitation fields become incoherent. More generally, we raise the question as to whether an ensemble of weakly correlated light fields such as those representing partially coherent wavefronts - can be protected in photonic topological insulators? Addressing this question is considerable importance as all optical fields undergo random fluctuations which may be small, as in many lasers, or large as in light generated by thermal sources.

Here, we investigate theoretically the the topological protection of partially coherent light propagating through disordered photonic topological insulators and uncover the aspects of light correlations that survive. This endeavor is appealing beyond the previously reported advantages of photonic topological insulators because it addresses the interplay between disorder in topological systems and disorder in the excitation field, and it opens exciting possibilities at the interface of topological physics and partial coherence, such as topologically protected transport in systems with random illumination.

\section{Results}

\section{Partially coherent light in disordered photonic topological insulators.}

To analyze the topological protection of partially coherent light, we consider a continuous set of light fields that range from the fully coherent to the fully incoherent limit. In practice, such partially coherent waves are generated in common light sources such as 
diode-pumped solid-state lasers, laser diodes, light emitting diodes, super luminescent light emitting diodes and micro light emitting diodes. These light sources exhibit different levels of coherence ranging from the solid-state lasers which are the most spatially coherent source to light emitting diodes which are the most incoherent ones ${ }^{23}$.

For concreteness, we perform our analysis using the archetypal Haldane model which exhibits all the essential features of topological insulators such as time reversal-broken symmetry $^{2}$. In optics, the Haldane model is implemented in a finite honeycomb lattice of waveguides ${ }^{4}$ where each site is coupled to its nearest neighbors by a real hopping parameter $\kappa_{1}$, and to its next-nearest neighbors by a complex parameter $\kappa_{2} \exp (i \phi)$, where $\phi$ is the Haldane flux ${ }^{2}$. The Haldane model exhibits two phases: the trivial phase when $\phi$ is equal to 0 or $\pi$, and the topological phase when $\phi \in(0, \pi)$. In the topological phase, the system supports edge states with propagation eigenvalues lying in the topological gap which is proportional to $\kappa_{2} \sin (\phi)$, reaching its maximum at $\phi=\pi / 2$. Hence, the coupling matrix elements of a disordered Haldane lattice are given by $H_{m, n}=\delta_{m, n} \Delta_{n}+\kappa_{1} \sum_{\langle j, k\rangle}\left(\delta_{n, j} \delta_{k, m}+\delta_{m, j} \delta_{n, k}\right)+i \kappa_{2} \sum_{\langle\langle j, k\rangle\rangle}\left(\delta_{n, j} \delta_{k, m}-\delta_{m, j} \delta_{n, k}\right)$, where we have chosen $\phi=\pi / 2$, the symbols \langle\rangle and $\langle\langle\rangle\rangle$ indicate summation over nearest and nextnearest-neighbor waveguides, respectively. The term $\delta_{m, n} \Delta_{n}$ represents the disordered on-site refractive indices which are generated using a truncated (at FWHM) normal distribution of unit width, zero mean, and scaled by the disorder strength parameter $\Delta$, Fig. (1-a). Furthermore, we use the particular (normalized) nearest-neighbor couplings $\kappa_{1}=1$ and next-nearest-neighbor couplings $\kappa_{2}=1 / 5$, such that the system exhibits edgemodes within the bulk-gap $\Delta_{b} \approx 2$, Fig. (1-a). Throughout this work we normalized the units in terms of $\kappa_{1}$.

For scalar wave fronts, a partially coherent wave is described by the mutual intensity function $\hat{\rho}\left(\boldsymbol{x}_{\mathbf{1}}, \boldsymbol{x}_{\mathbf{2}} ; z\right)=\left\langle E\left(\boldsymbol{x}_{\mathbf{1}}, z\right) E^{*}\left(\boldsymbol{x}_{\mathbf{2}}, z\right)\right\rangle^{24}$, where $E(\boldsymbol{x}, z)$ represents the scalar field along the transverse vector $\boldsymbol{x}$, and the angle bracket denotes time average or ensemble average, which is characterized by the coherence properties of the wavefront at the plane $z$. For linear waveguide lattices, the evolution of $\hat{\rho}\left(\boldsymbol{x}_{\mathbf{1}}, \boldsymbol{x}_{\mathbf{2}} ; z\right)$ is governed by the Liouville's equation

$$
i \frac{d \hat{\rho}}{d z}=\mathcal{L} \hat{\rho}
$$

where $\mathcal{L}=(\boldsymbol{H} \otimes \boldsymbol{I}-\boldsymbol{I} \otimes \boldsymbol{H})$. In addition, $\boldsymbol{H}$ is the lattice Hamiltonian (coupling matrix), $\boldsymbol{I}$ is the identity matrix of the same dimensions, and $\hat{\rho}$ is expressed as a vector. The solution to Eq. (1), written in matrix form, is given as $\hat{\rho}\left(\boldsymbol{x}_{\mathbf{1}}, \boldsymbol{x}_{\mathbf{2}} ; z\right)=U(z) \hat{\rho}\left(\boldsymbol{x}_{\mathbf{1}}, \boldsymbol{x}_{\mathbf{2}} ; 0\right) U^{\dagger}(z)$, where $U(z)=\exp (-i \boldsymbol{H} z)$ is the evolution operator.

Due to the anti-symmetric nature of $\mathcal{L}$, the eigenspectrum in Liouville space is given by all combinations $\Lambda_{m, n}=\lambda_{m}-\lambda_{n}$, where $\lambda_{n}$ are the eigenvalues of the Hamiltonian. 
a

Haldane lattice

Liouville eigenvalues $\lambda_{p}-\lambda_{q}$

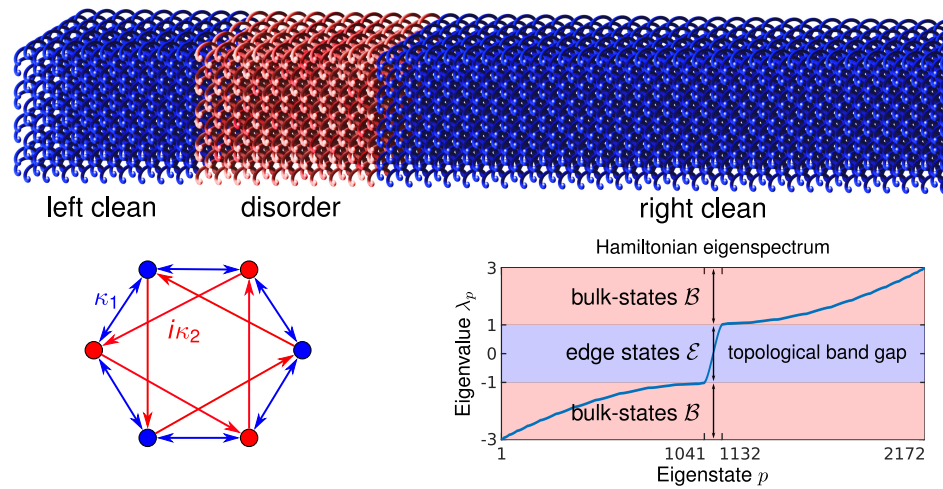

b

Topologically trivial 1D-lattice
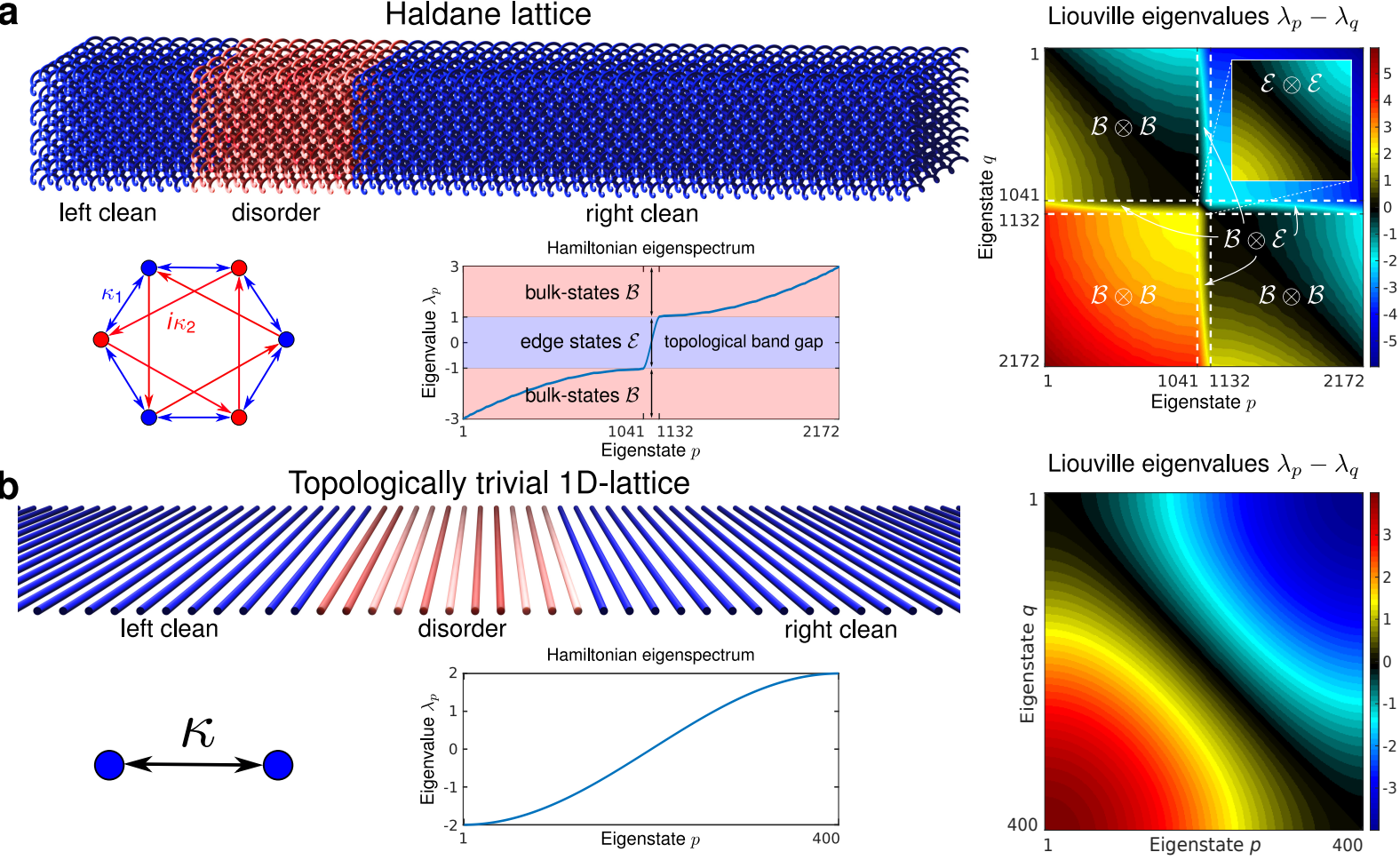

Figure 1. The Haldane lattice. a Photonic topological insulator implemented using a honeycomb lattice of helical waveguides with coupling coefficients as described in the hexagonal cell. For fully coherent light the eigenspectrum exhibits two regions $\mathcal{B}$ of bulk states and a gap crossed by the edge states $\mathcal{E}$. For our analysis we consider a lattice consisting of 11 hexagonal cells in the vertical direction and 90 in the horizontal, $\kappa_{1}=1$, and $\kappa_{2}=1 / 5$. b Trivial 1D lattice with couplings $\kappa$. In the absence of disorder, the discrete eigenspectrum exhibits a sinusoidal shape and the corresponding Liouville eigenvalues are degenerated. In both cases, the Liouville eigenspectrum is shown in the right column for the disorder-free systems. The disordered regions (red waveguides) encompass 20 waveguides in the horizontal direction, the initial states are launched in the left clean regions, and they propagate to the right.

Consequently, in Liouville space the characteristic band-gap of topological insulators does not exist. Instead, the spectrum exhibits massive degeneracies of edge-edge, edge-bulk, and bulk-bulk coherences, as indicated by the black diagonal region in right panel of Fig. (1-a).

In general, the mutual intensity $\hat{\rho}\left(\boldsymbol{x}_{\mathbf{1}}, \boldsymbol{x}_{\mathbf{2}} ; z\right)$ can be expressed as a superposition of coherent modes $\hat{\rho}\left(\boldsymbol{x}_{\mathbf{1}}, \boldsymbol{x}_{\mathbf{2}} ; z\right)=\sum_{k} \lambda_{k} \varphi_{k}^{*}\left(\boldsymbol{x}_{\mathbf{1}}\right) \varphi_{k}\left(\boldsymbol{x}_{\mathbf{2}}\right)$, where $\varphi_{k}(\boldsymbol{x})$ are appropriate eigenfunctions and $\lambda_{k}$ are the corresponding eigenvalues ${ }^{24}$. In this framework, a partially coherent field can be thought of as a superposition of spatially coherent but mutually uncorrelated modes $\varphi_{k}$ whose power is $\lambda_{k}$. In consequence, for conservative systems the eigenvalues $\lambda_{k}$ fulfill the condition $\sum_{n} \lambda_{n}=1$. The coherent mode representation allows us to define the square of the overall degree of coherence as $\mu^{2}=\sum_{n} \lambda_{n}^{2} /\left(\sum_{n} \lambda_{n}\right)^{2}=\sum_{n} \lambda_{n}^{225}$, whose inverse is referred to as the Schmidt number $S_{N}=1 / \mu^{226}$. This means that a fully coherent beam $\rho_{c}$ is comprised of a single spatial mode, whereas maximally incoherent fields $\rho_{i}$ are characterized by a uniformly weighted superposition of modes, $\rho_{i} \propto \boldsymbol{I}^{24}$, where $\boldsymbol{I}$ is 

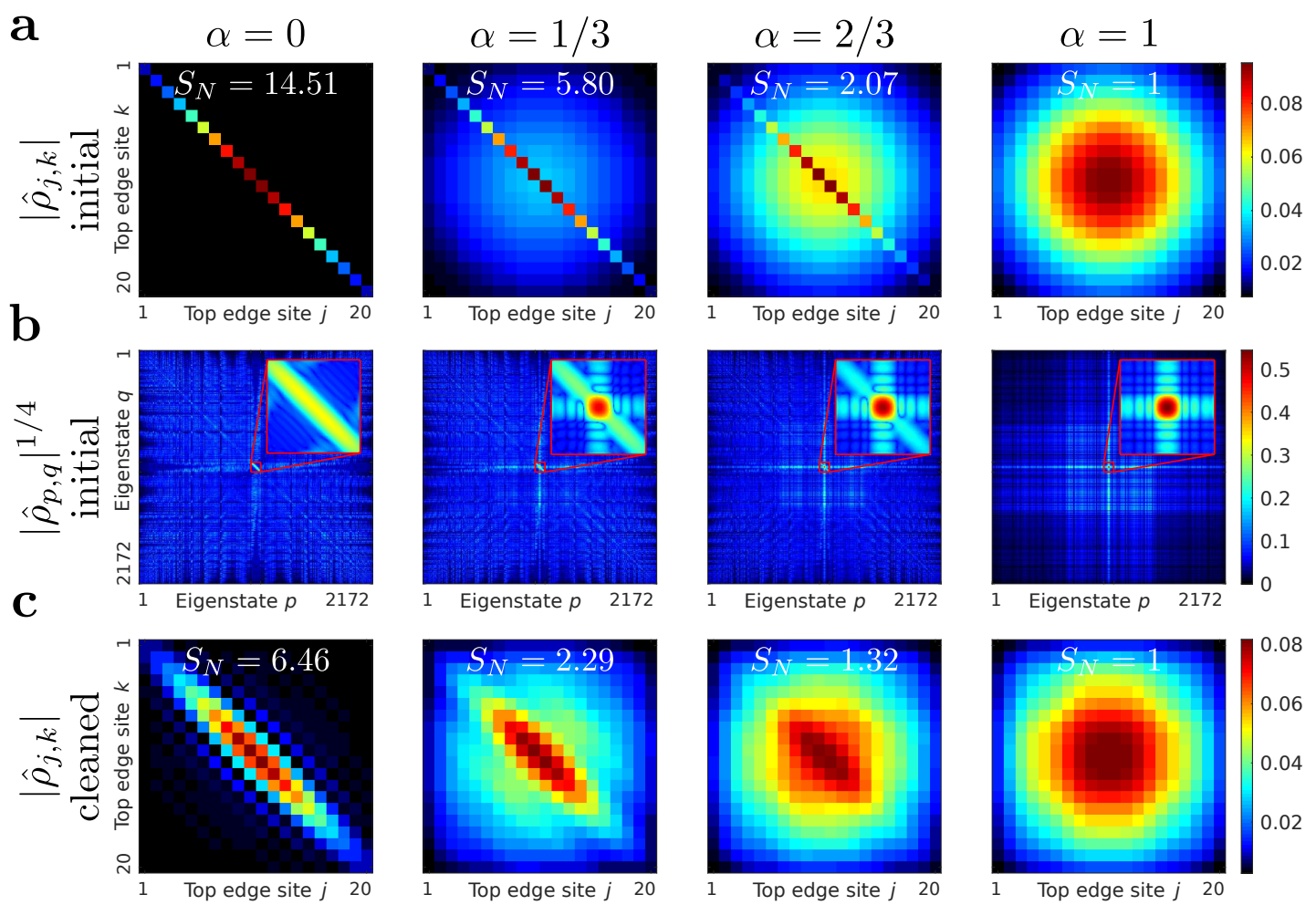

Figure 2. Initial light states. a and $\mathbf{b}$ show the absolute value of the spatial $\left|\hat{\rho}_{j, k}\right|$ and spectral $\left|\hat{\rho}_{p, q}\right|$ coherences, respectively, for some examples of initial states $\hat{\rho}_{\alpha}$ in the Haldane lattice. To improve the visibility of small components within the spectral edge-edge subspace, the insets in $\mathbf{b}$ depict $\sqrt[4]{\left|\hat{\rho}_{p, q}\right|}$. In $\mathbf{c}$ we show the spatial coherences $\left|\hat{\rho}_{j, k}\right|$ for the states obtained after removing all spectral bulk-bulk components. In all cases we observe significant changes in the shape of the spatial coherences, and in the most extreme case $(\alpha=0)$ the removal of the spectral bulk components transforms a formerly incoherent state (left-most panel in a) into a partially coherent one (left-most panel in c).

the identity matrix.

To construct the initial partially coherent fields we combine the coherent and incoherent extremes

$$
\hat{\rho}_{\alpha}=\alpha \hat{\rho}_{c}+(1-\alpha) \hat{\rho}_{i}
$$

where the real parameter $\alpha$ controls the degree of spatial coherence $(0 \leq \alpha \leq 1)$, with $\alpha=1(\alpha=0)$ corresponding to a fully coherent (incoherent) field. To generate $\hat{\rho}_{c}$ we use a discrete Gaussian function $\boldsymbol{E}$ with elements $E_{m}=A \exp (i m \phi) \exp \left(-(m-l)^{2} / 2 \sigma^{2}\right)$, such that $\hat{\rho}_{c}=\boldsymbol{E} \otimes \boldsymbol{E}^{\dagger}$, while $\hat{\rho}_{i}$ is the state obtained from $\hat{\rho}_{c}$ by deleting all off-diagonal elements to get $\left[\hat{\rho}_{i}\right]_{m, n}=\left|E_{m}\right|^{2} \delta_{m, n}$, see Fig. (2-a). Here, $A$ is a normalization constant, $\phi$ is an appropriate phase that imprints the proper momentum onto the wavepackets, $l$ is the spatial center, and $\sigma$ defines the Gaussian width.

In their present form, $\hat{\rho}_{c}$ and $\hat{\rho}_{i}$ have an eigenspectrum formed by all combinations of edge and bulk eigenmodes as illustrated in Fig. (2-b). However, to fully exploit topological protection, it is essential that the initial wave packets comprise only edge modes. To fulfill this requirement, we project $\hat{\rho}_{\alpha}$ onto the lattice eigenstates to obtain the spectral 
representation and set to zero all bulk-components, we then renormalize the resulting state and transform it back to the spatial representation. The absolute value of the resulting states are shown in Fig. (2-c). It is important to remark that the bulk-cleaning procedure renders states with a higher degree of coherence than their incoherent 'progenitors', yet the resulting states are never fully coherent. Further, the bulk-cleaning procedure leaves the spatial shape of fully coherent states practically unchanged, while partially coherent and fully incoherent states acquire significantly different spatial shapes, e.g. $\hat{\rho}_{i}$ which was fully diagonal now contains some coherences (off-diagonal elements) as shown in the leftmost panel of Fig. (2-c). The emergence of these coherences is our first important result, it clearly implies that to create wave packets comprising only edge-modes, it is required to meet a certain degree of spatial coherence. In what follows we use the bulk-free states as initial states.

In a fully coherent scenario, topological protection of the state $\hat{\rho}_{c}$ is manifests as unidirectional energy transport along the lattice's edges with only $1 \%$ of the total energy being scattered into the bulk or back-scattered by disorder, as depicted in Fig. (3-a). In contrast, for partially coherent light the disordered region acts as a barrier within which more and more light gets arrested as the degree of coherence decreases, as shown in Fig. (3-b).

To elucidate the impact of disorder on the topological protection of partially coherent light we examine two figures of merit, the transmittance and the fidelity. The transmittance is defined as the light intensity transferred through the disordered region, $T=\sum_{j}\left[\hat{\rho}_{\alpha}\left(z_{l}\right)\right]_{j, j}$, where $\hat{\rho}_{\alpha}$ are the evolved states in the disordered lattices, and the sum runs only over the sites in the region to the right of the disordered area. The fidelity, $F$, is computed between the states evolved in the disordered lattice $\hat{\rho}_{\alpha}\left(z_{l}\right)$, and the reference states $\hat{\rho}_{d f}\left(z_{f}\right)$ obtained after propagating the same initial state $\hat{\rho}_{\alpha}(0)$ through a disorder-free lattice. $F$ is given as $F\left(\hat{\rho}_{\alpha}\left(z_{l}\right), \hat{\rho}_{d f}\left(z_{f}\right)\right)=1-D\left(\hat{\rho}_{\alpha}\left(z_{l}\right), \hat{\rho}_{d f}\left(z_{f}\right)\right)$, where $D\left(\hat{\rho}_{\alpha}\left(z_{l}\right), \hat{\rho}_{d f}\left(z_{f}\right)\right)=\operatorname{Tr}\left(\left|\hat{\rho}_{\alpha}\left(z_{l}\right)-\hat{\rho}_{d f}\left(z_{f}\right)\right|\right) / 2$ is the trace distance which measures the overlap between the light states $\hat{\rho}_{\alpha}$ and $\hat{\rho}_{d f}{ }^{27}$. To be precise, $D=0$ iff $\hat{\rho}_{\alpha}=\hat{\rho}_{d f}$, and $(0<D \leq 1)$ stands for the opposite, $\hat{\rho}_{\alpha} \neq \hat{\rho}_{d f}$. In turn, a fidelity of $F=1$ attests that $\hat{\rho}_{\alpha}$ has not undergone any deviation from $\hat{\rho}_{d f}$, while $(0 \leq F<1)$ measures the degree of dissimilarity between both states. Indeed, $F=1$ is only obtained when both states traverse the same system. Note, $z_{f}$ is the propagation distance after which $\hat{\rho}_{d f}$ has reached the right side of the disorder-free system, that is, the right blue area in the Haldane lattice shown in Fig. (1-a). In the presence of disorder, the states tend to slow down inside the disorder-barrier, such that $\hat{\rho}_{\alpha}\left(z_{f}\right)$ lags behind $\hat{\rho}_{d f}\left(z_{f}\right)$. To compensate this delay, we let the states evolve a slightly larger propagation distance $z_{l} \in\left[z_{f}, z_{f}+10\right]$, such that $F\left(\hat{\rho}_{\alpha}\left(z_{l}\right), \hat{\rho}_{d f}\left(z_{f}\right)\right)$ is at a local maximum. Further, we only consider the transmitted part 


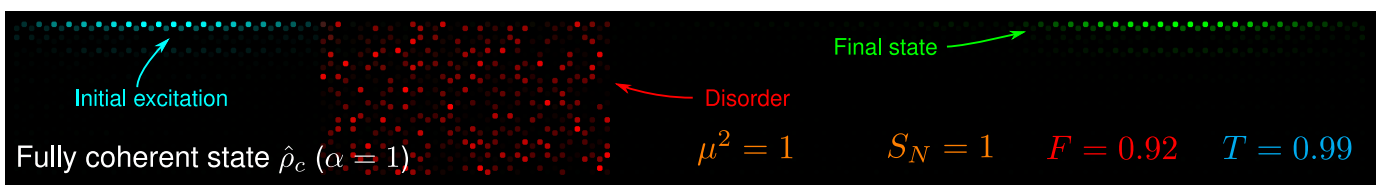

b

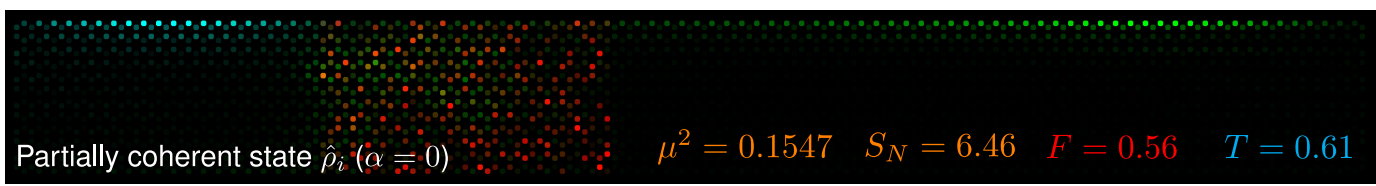

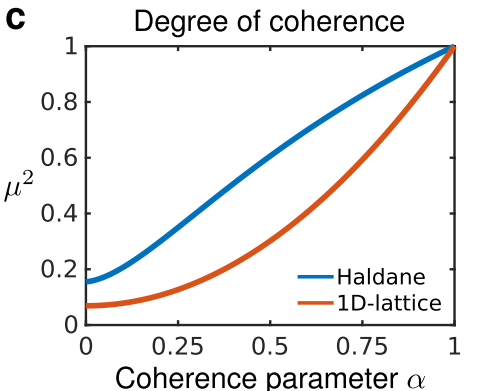

d

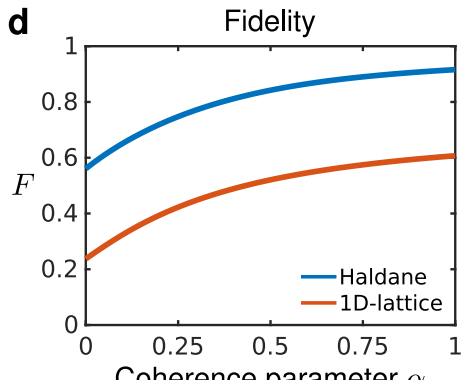

e

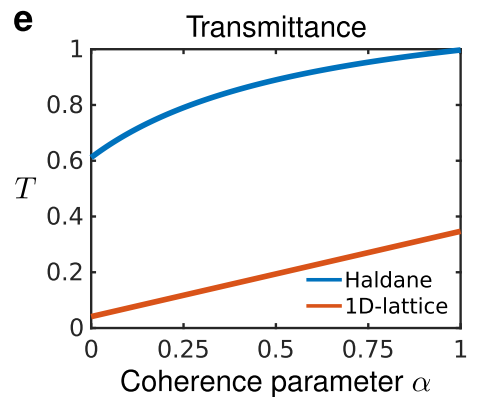

Figure 3. Topological protection of coherent and partially coherent light states. a and $\mathbf{b}$ depict the initial and final intensities of the coherent $\hat{\rho}_{c}$ and the incoherent $\hat{\rho}_{i}$ states, respectively. Both states are initialized with a Gaussian width $\sigma=6$ and the lattices exhibit a disorder strength $\Delta=1$. After a propagation distance of $z=75$ both states reach the right side of the lattice. While the fully coherent excitation freely passes through the disorder-barrier $\mathbf{a}$, the partially incoherent state scatters into the bulk b. In $\mathbf{c}, \mathbf{d}$, e we show the degree of coherence $\mu^{2}$, fidelities, and transmittances as a function of the coherence parameter $\alpha$ for the states $\hat{\rho}_{\alpha}$ in systems with disorder strength $\Delta=1$.

of $\hat{\rho}_{\alpha}\left(z_{l}\right)$ and $\hat{\rho}_{d f}\left(z_{f}\right)$ to compute the trace distance $D$, i.e., their projection onto the clean region to the right of the disorder-barrier.

To provide a first insight into the topological protection of partially coherent light, we consider the evolution of $\hat{\rho}_{\alpha}$ in systems with a relatively high disorder strength $\Delta=1$. As shown in Fig. $\left(3 \mathrm{c}\right.$ ), the degree of coherence $\mu^{2}$ for the trial states $\hat{\rho}_{\alpha}$ is an increasing function of $\alpha$ with an upper bound $\mu^{2}=1$ for the fully coherent case $(\alpha=1)$. For fully coherent states $(\alpha=1)$ the Haldane lattice allows for a fidelity $F \approx 0.9$, Fig. (3-e), and a nearly perfect transmittance $T \approx 0.99$, Fig. $(3-\mathrm{d})$. However, as the degree of coherence decreases $(\alpha \rightarrow 0)$ both figures of merit $T$ and $F$ drop to about 0.6, Fig. (3-e,f). In other words, even for the most incoherent states analyzed here, up to $60 \%$ of the total energy is topologically protected because it gets through the disordered barrier while $40 \%$ is scattered into the bulk.

To spotlight the advantages provided by topological protection, we have performed the corresponding analysis for a one-dimensional disordered lattice. The coupling matrix elements for this system are given by $H_{m, n}=\kappa\left(\delta_{m+1, n}+\delta_{m-1, n}\right)+\delta_{m, n} \Delta_{n}$, where $\kappa$ represents the coupling coefficients between nearest neighbor waveguides, and $\Delta_{n}$ represents the random on-site refractive index implemented in the same way as for the Haldane lattice, Fig. (1-b). In the absence of disorder, the spectrum of a 1D lattice comprising $M$ 
waveguides is analytically given as $\lambda_{n}=-2 \kappa \cos (\pi n /(M+1))$, which in Liouville space acquires the form $\Lambda_{p, q}=-2 \kappa \cos (\pi p /(M+1))+2 \kappa \cos ((\pi q /(M+1))$, Fig. (1-b). Notice, in this case the initial excitations are constructed in the same way as for the Haldane lattice, with the obvious exception of the bulk-cleaning procedure. Computation of the transmittance and fidelity reveals that in 1D lattices even fully coherent states cannot withstand the impact of disorder at the same level as the worst partially coherent case in the Haldane lattice: the best transmittance and fidelity for the 1D lattice are found to be $T \approx 0.38$ and $F \approx 0.6$ for fully coherent states, see Fig. (3-d,e).

We now examine the parameter regime within which partially coherent light experience topological protection. To do so, we compute the transmittances for states $\hat{\rho}_{\alpha}$ with degree of coherence $\alpha \in[0,1]$, traversing lattices with disorder strengths $\Delta \in[0,2]$. The results are summarized in Fig. (4). As clearly seen in Fig. (4 a), irrespective of the coherence parameter $\alpha$, partially coherent states endure much stronger disorder (see red area bounded by the contour line 0.9 in Fig. (4-a)), and they allow transmittances $T>0.9$ for disorder strengths as high as $\Delta=1.4$. Even more intriguingly, our estimates reveal that the most incoherent cases $(\alpha=0)$ exhibit transmittances $T>0.8$ for disorder strengths $\Delta \in[0,0.5]$. That is, even when the disorder closes the band-gap from $(-1,1)$, as indicated by the blue region in the spectrum of Fig. $(1-\mathrm{a})$, to $(-0.8,0.8)$ for $(\Delta=0.5)$, the most incoherent states show a considerable robustness to the impact of disorder.

To grasp these results we refer to the eigenspectra shown in the insets of Fig. (2-b) and notice that in the topological lattice, as the states become more incoherent they tend to populate edge-edge coherences that are spectrally close to the bulk-bulk and edge-bulk subspaces. As a result, any perturbation, or disorder, unavoidably induces substantial overlap of edge-edge and bulk-bulk coherences leading to localization of light into the disorder-barrier, thereby preventing energy transport through it. Correspondingly, for a disorder-free $1 \mathrm{D}$ lattice $(\Delta=0)$, we find that a minimum value of $\alpha \approx 0.8$ is required to obtain a transmittance of $T=0.9$, and $T>0.9$ can only be obtained for fully coherent states $(\alpha=1)$ and weak disorder $(\Delta<0.5)$. However, by gradually increasing the disorder strength, the transmittance quickly drops to 0 , Fig. (4-c). This poor performance in the transport of energy occurs because for $\alpha<1$ the light states suffer from back-scattering more prominently than the highly coherent ones $(\alpha=1)$. Clearly, the clean and disordered cases behave entirely differently; as a result of backscattering, partially coherent fields are destroyed in non-topological lattices, while in topological lattices partially coherent light still experience high degree of protection as described above.

In the presence of disorder, the spectral band-gap of a photonic topological insulator becomes narrower and this process allows the coupling between intermediate energy 


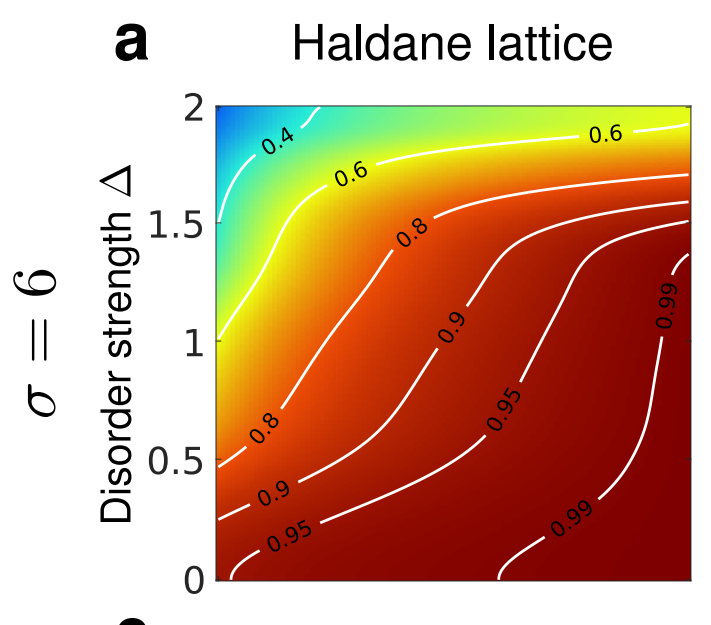

b

1D-lattice
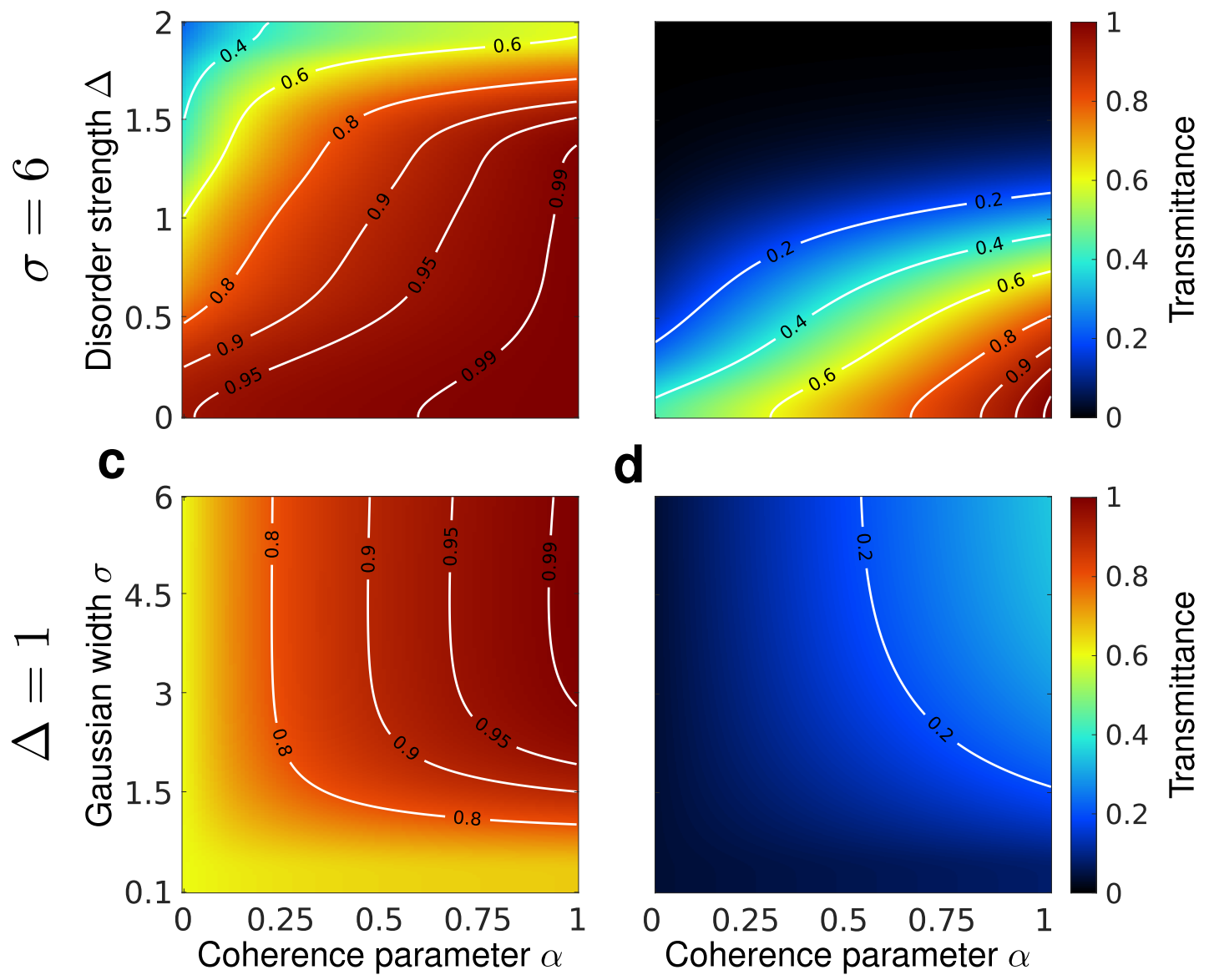

d

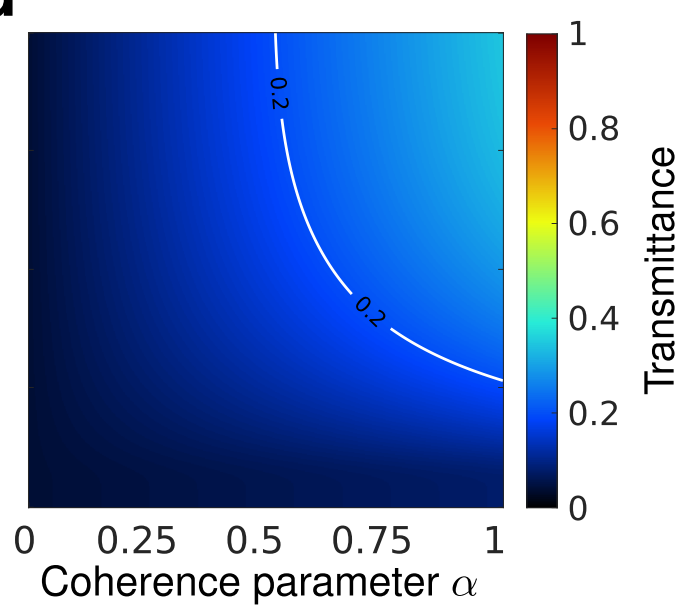

Figure 4. Transmittance versus disorder strength $\Delta$ and spatial widths $\sigma$ of the initial states. $\mathbf{a}$ and $\mathbf{b}$ depict the transmittance $T$ for the Haldane lattice and the 1D lattice versus the disorder strength $\Delta \in[0,2.5]$ and $\alpha$ for initial states with a Gaussian width $\sigma=6$. Similarly, c, $\mathbf{d}$ show $T$ as a function of $\alpha$ for initial excitations with spatial widths $\sigma \in[0.1,6]$ in lattices with disorder strength $\Delta=1$.

bulk-states and the edge-modes with the lowest and largest energy within the band gap. Such a coupling unavoidably increases as the spatial extension of the initial states becomes smaller. To quantify the effects of the corresponding disorder-induced coupling for partially coherent light, we consider light states exhibiting spatial widths in the range $\sigma \in[0.1,6]$ propagating in systems with relatively high disorder strength $\Delta=1$. We find that states with widths $\sigma \in[1.5,6]$ and coherence parameters $\alpha \in[0.25,1]$ achieve transmittances $T>0.8$, Fig. (4-c). Hence, we can define the threshold ( $\alpha=0.25, \sigma=1.5)$ above which partially coherent light presents the highest transmittances. For the 1D lattice, the width of the states plays no role and the transmittance remains below 0.4 in the whole range $\alpha \in[0,1]$ and $\sigma \in[0,6]$, Fig. (4-d).

We now study the fidelity as a function of the coherence parameter $\alpha \in[0,1]$ and the disorder strength $\Delta \in[0,2]$ for states $\hat{\rho}_{\alpha}$ with a fixed width $\sigma=6$. In agreement with the transmittance analysis, all the initial states render the same threshold $F \geq 0.8$ in the whole coherence interval $\alpha \in[0,1]$ for the same levels of disorder $\Delta \in[0,1]$, Fig. (5-b). 


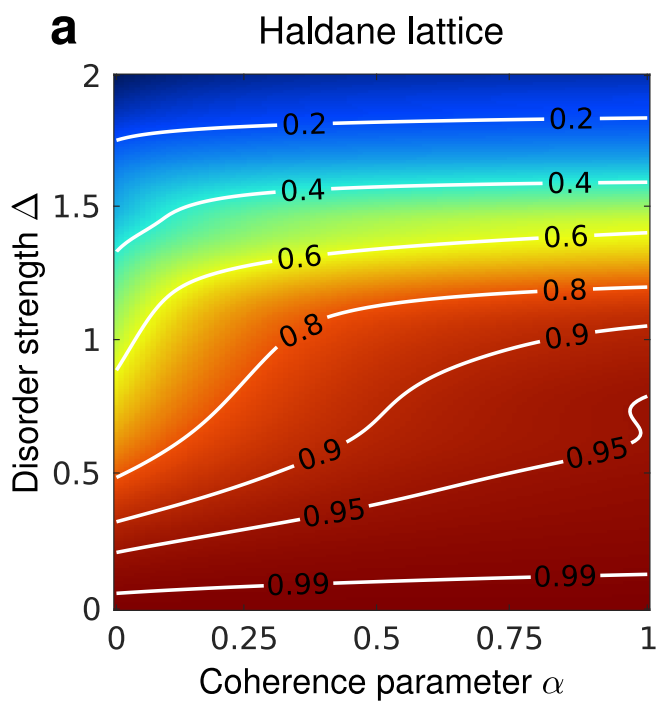

b 1D-lattice
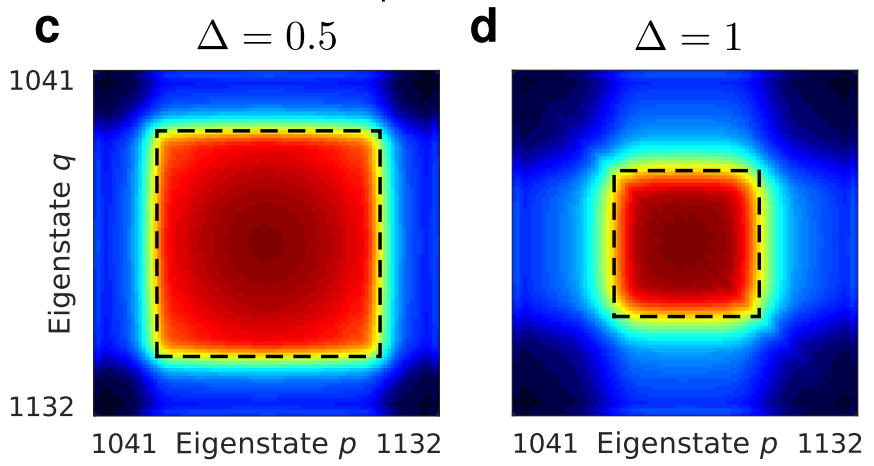

1041 Eigenstate $p 1132$

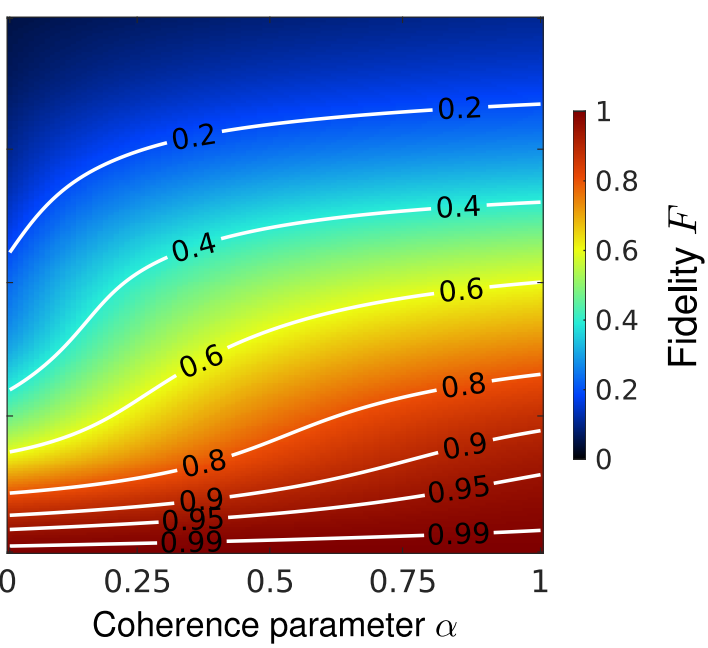

e $\Delta=1.5$

Figure 5. Fidelity and the topological window of protection. Parameter scans of the resulting transmitted fidelity $F$ for initial excitations with Gaussian spatial width $\sigma=6$ propagating in systems with disorder strength $\Delta \in[0,2.5]$ for the Haldane $\mathbf{a}$ and the $1 \mathrm{D}$ lattice $\mathbf{b}$. To identify the topological window of protection, we considered a spectrally broad (spatially narrow) partially coherent state with $\sigma=0.1$ as initial state and propagate it through an ensemble of 5000 random Haldane lattices. In disorder-free systems, the spectral correlation map remains intact. As the disorder strength increases, e.g. $\mathbf{c} \Delta=0.5, \mathbf{d} \Delta=1$, and $\mathbf{e} \Delta=0.5$, the band-gap reduces yielding to a reduction of the spectral window of protection as indicated by the dashed squares shown in the edge-edge subspace.

For the trivial $1 \mathrm{D}$ case, $F \geq 0.8$ is encountered for very weak disorder $\Delta \leq 0.5$, and the equality $\Delta=0.5$ only holds for fully coherent states. This clearly shows that the fidelity response of the $1 \mathrm{D}$ lattice is always outperformed by the photonic topological insulator.

\section{The topological window of protection.}

We have found that partially coherent light can be structured to possess relatively high topological immunity. Concomitantly with the drop in the degree of coherence, the corresponding eigenspectra extend over the bulk-bulk and edge-bulk subspaces causing rapid deterioration of the wave packets after propagating through disorder. Hence, the key to optimize topological protection for partially coherent light is to minimize the coupling induced by disorder of the initial spectrum with the edge-bulk and bulk-bulk spectral regions. This optimization can be achieved by first noting that for every instance of disor- 
der $\Delta$ there exists a different spectral window within which light states enjoy topological protection. To deduce the protection window we launch a spatially very narrow fully coherent bulk-cleaned excitation through an ensemble of $N=5000$ disordered Haldane lattices. In Fig. (5 c-e) we show the ensemble-average of the spectral coherences after propagation through disorder $\left\langle\left|\hat{\rho}_{p, q}\left(z_{f}\right)\right|\right\rangle_{N}$. In the disorder-free case $(\Delta=0)$ we observe that, as expected, the spectral coherences remain invariant, $\left\langle\left|\hat{\rho}_{p, q}\left(z_{f}\right)\right|\right\rangle_{N}=\left|\hat{\rho}_{p, q}(0)\right|$. One can see that the only surviving spectral intensities and coherences lie in a square region, the spectral window of topological protection, which shrinks with increasing levels of disorder. From these results it easy to see why even the bulk-cleaned states are not completely protected by topology. As shown in the insets of Fig. (2-b), when the coherence parameter $\alpha$ is below 1 the spectral coherences acquire an elongated elliptical shape in the edge-edge subspace. Depending on the strength of the disorder, the tails of the ellipse may fall outside of the window of protection facilitating the scattering into the bulk, resulting in reduced transmittance and fidelity.

\section{Conclusion}

We have demonstrated that partially coherent light survives randomness associated with disordered photonic topological insulators. This is in stark contrast with the case of nontopological systems where light excitations deteriorate very quickly when full coherence cannot be achieved. We have shown that the vulnerability of the partially coherent states is due to the inherent overlap of spectrum with the bulk-bulk and bulk-edge coherences. Hence, to grant topological protection to partially coherent light we have to keep the spectral coherence maps in the center of a well defined spectral window - the topological window of protection. This condition of course limits the degree of spatial coherence that guarantees robustness of light. Yet, it presents an important tool to define the precise coherence attributes of light in order to be protected in topological insulators. This is especially important, since in a real world scenario perfectly coherent light is unobtainable. Thus, our work is of considerable interest for the development of, for example, topological optical computing and information processing systems ${ }^{28,29}$. As we have shown, the fullcoherence condition for the initial light fields can be relaxed, and the advantages offered by topology can be enjoyed using only partially coherent beams. This opens up the design space to a wider selection of light sources, possibly yielding smaller, cheaper and more robust devices based on the topological transport of light.

* konrad.tschernig@physik.hu-berlin.de 
$\dagger$ bandres@creol.ucf.edu

‡ apleija@gmail.com

[1] Z. Wang, Y. Chong, J. D. Joannopoulos, and M. Soljačić, Observation of unidirectional backscattering-immune topological electromagnetic states, Nature 461, 772 (2009).

[2] F. D. M. Haldane, Model for a quantum hall effect without landau levels: Condensed-matter realization of the "parity anomaly", Phys. Rev. Lett. 61, 2015 (1988).

[3] C. He, H.-S. Lai, B. He, S.-Y. Yu, X. Xu, M.-H. Lu, and Y.-F. Chen, Acoustic analogues of three-dimensional topological insulators, Nature Communications 11, 2318 (2020).

[4] M. C. Rechtsman, J. M. Zeuner, Y. Plotnik, Y. Lumer, D. Podolsky, F. Dreisow, S. Nolte, M. Segev, and A. Szameit, Photonic floquet topological insulators, Nature 496, 196 (2013).

[5] A. B. Khanikaev, S. Hossein Mousavi, W.-K. Tse, M. Kargarian, A. H. MacDonald, and G. Shvets, Photonic topological insulators, Nature Materials 12, 233 (2013).

[6] M. Hafezi, S. Mittal, J. Fan, A. Migdall, and J. M. Taylor, Imaging topological edge states in silicon photonics, Nature Photonics 7, 1001 (2013).

[7] K. Fang, Z. Yu, and S. Fan, Realizing effective magnetic field for photons by controlling the phase of dynamic modulation, Nature Photonics 6, 782 (2012).

[8] M. Hafezi, E. A. Demler, M. D. Lukin, and J. M. Taylor, Robust optical delay lines with topological protection, Nature Physics 7, 907 (2011).

[9] Y. Poo, R.-x. Wu, Z. Lin, Y. Yang, and C. T. Chan, Experimental realization of self-guiding unidirectional electromagnetic edge states, Phys. Rev. Lett. 106, 093903 (2011).

[10] X. Zhang, M. Xiao, Y. Cheng, M.-H. Lu, and J. Christensen, Topological sound, Communications Physics 1, 97 (2018).

[11] Z. Yang, F. Gao, X. Shi, X. Lin, Z. Gao, Y. Chong, and B. Zhang, Topological acoustics, Phys. Rev. Lett. 114, 114301 (2015).

[12] L. Lu, J. D. Joannopoulos, and M. Soljačić, Topological photonics, Nature Photonics 8, 821 (2014).

[13] M. A. Bandres, S. Wittek, G. Harari, M. Parto, J. Ren, M. Segev, D. N. Christodoulides, and M. Khajavikhan, Topological insulator laser: Experiments, Science 359, 10.1126/science.aar4005 (2018).

[14] G. Harari, M. A. Bandres, Y. Lumer, M. C. Rechtsman, Y. D. Chong, M. Khajavikhan, D. N. Christodoulides, and M. Segev, Topological insulator laser: Theory, Science 359, 10.1126/science.aar4003 (2018).

[15] M. A. Bandres, M. C. Rechtsman, and M. Segev, Topological photonic quasicrystals: Fractal topological spectrum and protected transport, Phys. Rev. X 6, 011016 (2016).

[16] S. Stützer, Y. Plotnik, Y. Lumer, P. Titum, N. H. Lindner, M. Segev, M. C. Rechtsman, 
and A. Szameit, Photonic topological anderson insulators, Nature 560, 461 (2018).

[17] Z. Yang, E. Lustig, Y. Lumer, and M. Segev, Photonic floquet topological insulators in a fractal lattice, Light: Science \& Applications 9, 128 (2020).

[18] M. C. Rechtsman, Y. Lumer, Y. Plotnik, A. Perez-Leija, A. Szameit, and M. Segev, Topological protection of photonic path entanglement, Optica 3, 925 (2016).

[19] S. Mittal, V. V. Orre, and M. Hafezi, Topologically robust transport of entangled photons in a 2d photonic system, Opt. Express 24, 15631 (2016).

[20] A. Blanco-Redondo, B. Bell, D. Oren, B. J. Eggleton, and M. Segev, Topological protection of biphoton states, Science 362, 568 (2018).

[21] K. Tschernig, Á. Jimenez-Galán, D. N. Christodoulides, M. Ivanov, K. Busch, M. A. Bandres, and A. Perez-Leija, Topological protection versus degree of entanglement of twophoton light in photonic topological insulators, Nature Communications 12, 1974 (2021).

[22] K. Tschernig, R. L. Franco, M. Ivanov, M. A. Bandres, K. Busch, and A. Perez-Leija, Topological protection of highly entangled non-gaussian two-photon states, Materials for Quantum Technology 1, 035001 (2021).

[23] Y. Deng and D. Chu, Coherence properties of different light sources and their effect on the image sharpness and speckle of holographic displays, Scientific Reports 7, 5893 (2017).

[24] E. Wolf, Introduction to the Theory of Coherence and Polarization of Light (Cambridge Univ. Press, 2007).

[25] M. Alonso, T. Setälä, and A. T. Friberg, Minimum uncertainty solutions for partially coherent fields and quantum mixed states, New Journal of Physics 16, 123023 (2014).

[26] J. Sperling and W. Vogel, The schmidt number as a universal entanglement measure, Physica Scripta 83, 045002 (2011).

[27] A. Smirne, S. Cialdi, G. Anelli, M. G. A. Paris, and B. Vacchini, Quantum probes to experimentally assess correlations in a composite system, Phys. Rev. A 88, 012108 (2013).

[28] M. Makwana, R. Craster, and S. Guenneau, Topological beam-splitting in photonic crystals, Opt. Express 27, 16088 (2019).

[29] Y.-F. Gao, J.-P. Sun, N. Xu, Z. Jiang, Q.-C. Hou, H. Song, M.-C. Jin, and C. Zhang, Manipulation of topological beam splitter based on honeycomb photonic crystals, Optics Communications 483, 126646 (2021). 